doi: $10.2306 /$ scienceasia1513-1874.2014.40.400

\title{
Distinctive roles of human Argonaute1, 2, 3, and 4 proteins on LINE-1 methylation and regulation of intragenic LINE-1-containing genes
}

\author{
Piyapat Pin-on $^{\mathrm{a}}$, Apiwat Mutirangura ${ }^{\mathrm{b}, *}$ \\ a Inter-department Program of Biomedical Sciences, Faculty of Graduate School, Chulalongkorn University, \\ Bangkok 10330 Thailand \\ b Centre of Excellence in Molecular Genetics of Cancer and Human Diseases, Department of Anatomy, \\ Faculty of Medicine, Chulalongkorn University, Bangkok 10330 Thailand
}

*Corresponding author, e-mail: mapiwat@chula.ac.th

Received 22 Aug 2014

Accepted 2 Dec 2014

\begin{abstract}
Argonaute proteins (AGOs) are evolutionarily conserved and ubiquitously expressed in all higher eukaryotes. They participate in cell differentiation, transposon silencing, and other important functions. AGOs play key roles in the genesilencing pathways guided by small RNAs. There are many human AGOs. However, redundancy and the distinctive roles of different AGOs have not been well characterized. Previously, we demonstrated that AGO2 down-regulates the expression of genes containing hypomethylated long interspersed nuclear elements (LINE-1s). Here, we evaluate the expression of genes containing LINE-1 in AGO1-4-knocked down HEK293 cells. Furthermore, we measured the methylation levels of AGO1-4-bound LINE-1s. Genes containing LINE-1s in AGO1, -2, -3 or -4-knocked down HEK293 cells showed prevented up-regulation, increased up-regulation, unchanged regulation and prevented up-regulation, respectively. Interestingly, AGO1-4 bound to LINE-1 differently in terms of the methylation level. Although the methylation level of AGO1 and -4-bound LINE-1s was not different from that of the genome, AGO2- and AGO3-bound LINE-1s were hypomethylated. Our experiments demonstrate the distinctive epigenetic roles of AGO1-4 in regulating genes containing LINE-1s.
\end{abstract}

KEYWORDS: DNA methylation, chromatin immunoprecipitation, transposon silencing, CU-DREAM, microarrays X

\section{INTRODUCTION}

It is well known that Argonaute proteins (AGOs) play a role in controlling gene expression. There are $8 \mathrm{AGO}$ genes in humans ${ }^{1,2}$, but there is limited information demonstrating whether the functions of each AGO protein are redundant or different ${ }^{3}$. $A G O$ genes are a highly conserved and extensively expressed in many organisms, including plants, animals, and humans ${ }^{4}$. There are many reports that human AGO proteins are involved in organ growth and development ${ }^{5}$, and recent studies have clarified its role as a potential factor related to oncogenesis in colonic, mammary, and prostate tissue ${ }^{6-8}$. Furthermore, several works have revealed that AGO1 is associated with pretranscriptional gene silencing. First, Ago1 in S. pombe is used to direct histone 3 lysine- 9 methylation (H3K9) of local transposon sequences, resulting in nucleosome compaction and transposon silencing; RNA polymerase II (RNAPII) activity is thus restricted at the targeted locus, followed by heterochromatin formation ${ }^{9}$. Second, Drosophila Ago1 also has a role in heterochromatin formation: the Ago1 mutant disrupts pre-transcriptional gene silencing of transgene tandem repeats, with the loss of $\mathrm{H} 3 \mathrm{mK} 9$ and silencing of heterochromatin ${ }^{10}$. Third, in A. thaliana, Ago1 may be the slicer component of RNA-induced silencing complex (RISC), acting as an RNA slicer that uses the sequence of an associated siRNAs to guide the cleavage of homologous RNAs in RISCs and in the maintenance of chromatin modifications at some loci. Furthermore, knockdown of Ago1 has been reported to be associated with developmental abnormalities ${ }^{11}$. In addition, Ago1 has been implicated in the silencing activity of promoter-targeted small RNAs. DNA methyltransferase (DNMT3a) has been shown to coimmunoprecipitate with small RNAs at the promoters of some targeted genes. One known mechanism is RNA interference, with Ago2 acting as a trans-acting element to down-regulate gene expression by binding to different classes of small noncoding RNAs, including microRNAs (miRNAs) and small interfering RNAs (siRNAs), which guide proteins to their specific targets via sequence complementarity ${ }^{12-14}$. Another 
mechanism is found in plants and is termed RNAdirected DNA methylation (RdDM). Ago4 is associated with siRNA, and the ribonucleoprotein complex is loaded onto chromatin via complementarity. In gene silencing, the complex guides methyltransferase enzymes to establish sequence-specific de novo DNA methylation ${ }^{15}$.

Recently, we reported that $\mathrm{AGO} 2$ down-regulates genes containing hypomethylated intragenic long interspersed elements-1 (LINE-1) ${ }^{16}$. LINE-1s comprise a group of abundant retrotransposon sequences found in large numbers in the human genome. Moreover, our follow-up report demonstrated that intragenic LINE-1 sequences are conserved and regulate several biological processes, including DNA damage and repair, inflammation, immune function, and cell differentiation. We screened for genes that are involved in intragenic LINE-1 regulation networks using a bioinformatics approach and found that intragenic LINE-1 acts as a cis-regulatory element within genes to modulate host gene expression. Furthermore, AGOs are also transacting elements and coordinate with LINE-1 to regulate gene expression ${ }^{17}$. For example, genes containing LINE-1 are up-regulated in si-AGO2 embryonic kidney cell lines. These results demonstrate that AGO2 plays a role in the control of gene expression for thousands of genes containing LINE-1s. Herein, we investigate whether the other AGOs serve as transacting elements that mediate gene expression through intragenic LINE-1 elements.

AGO1-4 plays a distinctive role in the control gene expression through upstream mononucleotide A-repeats ${ }^{3}$. We found that all members of the AGO family preferentially bind to A-repeats. AGO1-4 knockdown however produced different patterns. AGO1 up-regulated genes containing upstream A-repeats, whereas AGO2 and $\mathrm{AGO} 3$ were significantly associated with down-regulated genes containing upstream A-repeats. AGO4 did not significantly regulate gene expression.

However, it is unclear whether AGOs are associated with intragenic LINE-1 methylation and whether they lead to gene up- and/or down-regulation. Thus in this study, we evaluated the role of AGO1-4 in affecting the expression of genes containing LINE-1 using a bioinformatics approach. The mRNA levels in the HEK293 cell line knocked down for AGO1-4 were compared with intragenic LINE-1 genes by the connection up- and down-regulation expression analysis of microarrays (CU-DREAM) software package (http://pioneer.netserv.chula.ac.th/ achatcha/ cu-dream/ $/{ }^{18}$. This software calculates various statistical parameters, including Student's $t$-test and Pear- son's chi-squared test, to analyse the gene regulatory functions of intragenic LINE-1. To explore the association between AGOs and intragenic LINE-1 methylation, we performed chromatin immunoprecipitation (ChIP) and quantitative combined bisulphite restriction analysis of methylation pattern (Q-COBRA-MP) assays ${ }^{19}$.

\section{MATERIALS AND METHODS}

\section{Cell culture}

HEK293 cells were maintained in Dulbecco's modified Eagle's medium (Gibco BRL, Life Technologies) supplemented with $2 \mathrm{mM} \mathrm{L}$-glutamine, $10 \%$ (v/v) heat-inactivated foetal bovine serum (Sigma), $10 \mathrm{mg} / \mathrm{ml}$ antibiotic/antimycotic (Gibco BRL, Life Technologies) at $37^{\circ} \mathrm{C}$ and $5 \%(\mathrm{v} / \mathrm{v}) \mathrm{CO}_{2}$. The medium was changed 3 days later. The cells were harvested for the ChIP assay on the 7th day.

\section{Chromatin immunoprecipitation}

A ChIP analysis was performed according to a previously published protocol ${ }^{20}$. Briefly, $1 \times 10^{6}$ HEK293 cells in a $75-\mathrm{cm}^{2}$ flask were grown to $80 \%$ confluence. The cells were harvested, and formaldehyde was added at a final concentration of $1 \%$ directly to the cell culture medium. Fixation was performed at $37^{\circ} \mathrm{C}$ for $10 \mathrm{~min}$ and stopped by the addition of glycine to a final concentration of $0.125 \mathrm{M}$. As much medium as possible was removed, and the cells were washed twice with ice-cold PBS containing protease inhibitors; and then scraped into a conical tube. The cells were collected by centrifugation and rinsed in cold phosphate-buffered saline. The cell pellets were resuspended in lysis buffer $(0.5 \% \mathrm{NP} 40,85 \mathrm{mM}$ $\mathrm{KCl}, 5 \mathrm{mM}$ PIPES, $\mathrm{pH}$ 8.0), incubated on ice for $20 \mathrm{~min}$, and homogenized. The nuclei were collected by microcentrifugation and then resuspended in lysis buffer (1\% SDS, $10 \mathrm{mM}$ EDTA, $50 \mathrm{mM}$ Tris-HCl, $\mathrm{pH}$ 8.1) and incubated on ice for $10 \mathrm{~min}$. The samples were sonicated on ice with an Ultrasonics sonicator at setting 10 amplitude for $20 \mathrm{~s}$ to achieve an average length of approximately $200-1000 \mathrm{bp}$; the samples were then microcentrifuged. The sonicated cell pellet was resuspended 10-fold in ChIP dilution buffer with protease inhibitors. The fragment solution was precleared with the addition of protein A-positive cells for $15 \mathrm{~min}$ at $4{ }^{\circ} \mathrm{C}$. The precleared chromatin was incubated with $1 \mu \mathrm{g}$ of affinity-purified goat monoclonal antibody or no antibody and rotated at $4{ }^{\circ} \mathrm{C}$ for approximately $12-16 \mathrm{~h}$. The antibodies used included EIF2C1-4 (anti-AGO1-4) or a control non-immunized goat antibody. Protein A agarose 
Table 1 Gene grouping according to presence of LINE-1 elements and regulated expression.

\begin{tabular}{lcc}
\hline & Up- or down-regulated genes of experiment & Not up- and not down-regulated genes of experiment \\
\hline Genes containing LINE-1 & Number of genes in group A & Number of genes in group B \\
Genes without LINE-1 & Number of genes in group C & Number of genes in group D \\
\hline
\end{tabular}

$(40 \mu \mathrm{l})$ was added for $2 \mathrm{~h}$ at $4{ }^{\circ} \mathrm{C}$ with rotation to collect the antibody/protein complex, and the complexes were washed and eluted. The pellet was gently centrifugation $\left(1084 g\right.$ at $\left.4{ }^{\circ} \mathrm{C}, 1 \mathrm{~min}\right)$, and the protein A agarose/antibody/protein complex was washed for 3-5 min on a rotating platform with each of the following buffers in order: (a) low-salt immune complex wash buffer $(1 \times 1 \mathrm{ml})$; (b) high-salt immune complex wash buffer $(1 \times 1 \mathrm{ml})$ and $(\mathrm{c}) \mathrm{LiCl}$ immune complex wash buffer $(1 \times 1 \mathrm{ml})$. The complex was eluted with elution buffer and shaken for $15 \mathrm{~min}$. The crosslinks were reversed by the addition of $\mathrm{NaCl}$ to a final concentration of $200 \mathrm{mM}$, and RNA was removed by the addition of $10 \mu \mathrm{g}$ of RNase A per sample, followed by incubation at $65^{\circ} \mathrm{C}$ for $4 \mathrm{~h}$. The samples were then precipitated at $-20^{\circ} \mathrm{C}$ overnight by the addition of 2.5 volumes of ethanol and then pelleted by microcentrifugation. The samples were resuspended in $100 \mu \mathrm{l}$ of Tris-EDTA ( $\mathrm{pH} 7.5$ ), $25 \mu \mathrm{l}$ of proteinase $\mathrm{K}$ buffer (1\% sodium dodecyl sulphate, $50 \mathrm{mM}$ Tris ( $\mathrm{pH} 7.5), 25 \mathrm{mM}$ EDTA) and $1.5 \mu$ l of proteinase $\mathrm{K}$ (Sigma) and incubated at $45^{\circ} \mathrm{C}$ for $2 \mathrm{~h}$. The samples were extracted with phenol-chloroform-isoamyl alcohol (25:24:1) followed by extraction with chloroformisoamyl alcohol and then precipitated with $1 / 10$ volume of $3 \mathrm{M} \mathrm{NaOAc}$ ( $\mathrm{pH} 5.3$ ), $5 \mu \mathrm{g}$ of glycogen and 2.5 volumes of ethanol. The pellets were collected by microcentrifugation and resuspended in $30 \mu \mathrm{l}$ of $\mathrm{H}_{2} \mathrm{O}$.

\section{Q-COBRA-MP}

The immunoprecipitated samples were used for bisulphite modification, as previously described ${ }^{19}$. The bisulphite-treated DNA $(2 \mu \mathrm{l})$ was amplified using COBRA LINE-1 primers. The reactions were incubated at $95^{\circ} \mathrm{C}$ for $10 \mathrm{~min}$, followed by 35 cycles of $95^{\circ} \mathrm{C}$ for $30 \mathrm{~s}, 54{ }^{\circ} \mathrm{C}$ for $30 \mathrm{~s}$, and $72{ }^{\circ} \mathrm{C}$ for $30 \mathrm{~s}$, with a final extension at $72{ }^{\circ} \mathrm{C}$ for $7 \mathrm{~min}$. The amplicons were digested in 10-ml reaction volumes with $2 \mathrm{U}$ of TaqI in $1 \times$ TaqI buffer (MBI Fermentas) at $65^{\circ} \mathrm{C}$ overnight and then electrophoresed through $8 \%$ non-denaturing polyacrylamide gels. The intensities of DNA fragments were measured with a PhosphorImager and analysed using MS EXCEL. The yields of methylated amplicons (TaqI positive) and unmethylated amplicons (uncut amplicon) were calculated as a percentage (the intensity of the methylated candidate gene digested by TaqI divided by the sum of the unmethylated amplicon and the TaqI-positive amplicons).

\section{Expression analysis}

To evaluate whether intragenic LINE-1 can control host gene expression through AGOs, we collected mRNAs of GSE4246 from the GEO database ${ }^{21}$ and prepared templates of the microarray, series matrix file, and platform. Each gene was compared to the means of the experimental and control groups and classified as up- or down-regulated and not upand not down-regulated, depending on the statistical significance of Student's $t$-test. Subsequently, the distributions of up- or down-regulated genes were evaluated as to whether the distributions were dependent on containing an intragenic LINE-1 using Pearson's chi-squared test. A list of genes containing LINE-1s has been reported ${ }^{17}$. Genes were grouped into four groups, A through D. The significantly up- or down-regulated genes containing intragenic LINE-1 were included in group A. The significant not upand not down-regulated genes containing intragenic LINE-1 were included in group B. The significant upor down-regulated genes without intragenic LINE-1 were included in group $\mathrm{C}$. The remaining genes (nonsignificant genes without LINE-1) were included in group $\mathrm{D}$ (Table 1). The $p$-values of the odds ratio (ORs), $p$-values and lower and upper $95 \%$ confidence intervals (CI) of the genes in groups A through D were displayed in an MS EXCEL format. All the statistical analyses were performed using extensions in the CU-DREAM software (http://pioneer.netserv. chula.ac.th/ achatcha/cu-dream/).

\section{RESULTS}

In this study, we determined whether the roles of human AGO1-4 in the epigenetic regulation of genes containing LINE-1 are different. The mRNA profiles of HEK293 cells knocked down for AGOs from GSE4246 was categorized as up- or down-regulated and not up- or not down-regulated; the statistical significance of 1454 genes containing or not intragenic LINE-1 was determined using Student's $t$-test and the chi-squared test. The results showed that the reduction of AGO2 expression in HEK293 cells significantly promoted the up-regulation of genes containing intragenic LINE-1, OR $=1.88, p$-value $=$ 
Table 2 Grouping of up- and down-regulated gene expression determined by CU-DREAM-X.

\begin{tabular}{|c|c|c|c|c|c|c|c|c|c|c|c|c|c|}
\hline & & \multicolumn{3}{|c|}{ Si-AGO1 } & \multicolumn{3}{|c|}{$\mathrm{Si}-\mathrm{AGO} 2$} & \multicolumn{3}{|c|}{$\mathrm{Si}-\mathrm{AGO} 3$} & \multicolumn{3}{|c|}{ Si-AGO4 } \\
\hline & & OR & $p$-value & $95 \% \mathrm{CI}$ & OR & $p$-value & $95 \% \mathrm{CI}$ & OR & $p$-value & $95 \% \mathrm{CI}$ & OR & $p$-value & $95 \% \mathrm{CI}$ \\
\hline up & LINE-1 & 0.55 & $4.67 \times 10^{-4}$ & $0.39-0.77$ & 1.88 & $6.19 \times 10^{-4}$ & $1.30-2.70$ & 0.78 & $4.45 \times 10^{-1}$ & $0.41-1.48$ & 0.33 & $1.34 \times 10^{-8}$ & $0.22-0.50$ \\
\hline down & LINE-1 & 1.29 & $5.21 \times 10^{-2}$ & $1.00-1.67$ & 0.82 & $4.57 \times 10^{-1}$ & $0.48-1.39$ & 1.29 & $3.03 \times 10^{-1}$ & $0.79-2.11$ & 1.27 & $7.99 \times 10^{-2}$ & $0.97-1.66$ \\
\hline
\end{tabular}

$6.19 \times 10^{-4}$ and $95 \% \mathrm{CI}=1.30-2.70$. This result supports that the biological function of $\mathrm{AGO} 2$ is to down-regulate genes containing intragenic LINE-1. In contrast, HEK293 cells knocked down for AGO3 did not show regulated gene expression. HEK293 cells knocked down for AGO1 and -4 showed hampered up-regulation, with significant ORs $=0.55$ and 0.33 , $p$-values $=4.67 \times 10^{-4}$ and $1.34 \times 10^{-8}, 95 \% \mathrm{CI}=$ 0.39-0.77 and 0.22-0.50, respectively. Conversely, AGO1-4 siRNA did not significantly alter downregulating genes containing LINE-1 elements. These results support the notion that $\mathrm{AGO} 1$ and $\mathrm{AGO} 4$ play a role that is opposite to AGO2 in terms of the expression of genes containing intragenic LINE-1 (Table 2).

To evaluate whether the epigenetic modification of AGOs bound to LINE-1s are different, we investigated the methylation status of LINE-1-bound AGO1-4 proteins by ChIP and Q-COBRA-MP. The results revealed that $\mathrm{AGO} 1$ and $\mathrm{AGO} 4$ preferentially bind to normally methylated LINE-1s, whereas AGO2 and $\mathrm{AGO} 3$ preferentially bind to hypomethylated LINE-1s (Fig. 1).

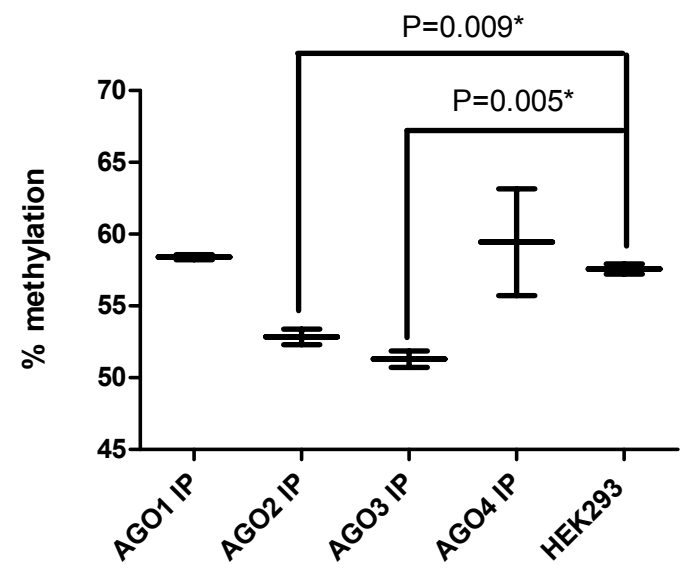

Fig. 1 ChIP and Q-COBRA-MP. Methylation percentage of AGO1 and AGO4 shows normality compared with the HEK293 cell line, whereas the methylation percentage of AGO2 and AGO3 shows hypomethylation compared with the HEK293 cell line.

\section{DISCUSSION}

Previously, we demonstrated that $\mathrm{AGO} 2$ represses genes containing hypomethylated LINE-1s. Here, we demonstrate that $\mathrm{AGO} 1$ and $\mathrm{AGO} 4$ regulate genes containing LINE-1s in an opposite manner as that of AGO2. Furthermore, AGO1 and AGO4 bind to different LINE-1 loci from AGO2. Whereas AGO2 and AGO3 bind to hypomethylated LINE1s, AGO1 and AGO4 bind to LINE-1s that have methylation levels similar to the entire genome.

The AGO2-mediated repression of genes containing hypomethylated LINE-1s is mediated by LINE-1 RNA. When intragenic LINE-1s are hypomethylated, LINE-1 RNA is transcribed; the LINE-1 RNA then forms a complex with pre mRNA and AGO2, resulting in mRNA depletion. The down-regulation of AGO1 and AGO4 prevents the up-regulation of expression. It is possible that AGO1 and AGO4 prevent LINE-1 transcription. Consequently, the presence of AGO1 and AGO4 at normally methylated LINE-1s prevents intragenic LINE-1 RNA production, and AGO1 and AGO4 consequently limit LINE-1 RNA from interfering with gene transcription. AGO3 binds to hypomethylated LINE-1s, similar to AGO2. Nevertheless, the depletion of AGO3 did not alter gene expression. The similar roles between $\mathrm{AGO} 2$ and $\mathrm{AGO} 3$ and the opposite roles between AGO1 and AGO2 were demonstrated previously with regard to the regulatory roles of mononucleotide $\mathrm{A}$ repeats ${ }^{3}$. We speculate that the $\mathrm{AGO} 2$ and $\mathrm{AGO} 3$ functions are redundant; thus the depletion of $\mathrm{AGO} 3$ was compensated for by AGO2.

In a variety of living organisms, AGO1 has the function of promoting heterochromatin; in plants, AGO4 promotes DNA methylation. It is possible that AGO1 and AGO4 in humans may be associated with heterochromatin and methylationassociated LINE-1s, with AGO1 and AGO4 binding to transcription-inactiveLINE-1s.

In conclusion, we explored whether AGO proteins play a different role in controlling gene expression. AGO2 represses genes containing hypomethylated LINE-1. AGO3 may be redundant to AGO2, while AGO2 plays a major role. The AGO1 and AGO4 roles are distinctive from $\mathrm{AGO} 2$ and have opposite 
effects in regulating gene expression. It is interesting to further explore the mechanisms underlying these differences.

Acknowledgements: This study has been supported by the Ratchadapiseksomphot Endowment Fund of Chulalongkorn University (CU-57-001-HR). Piyapat Pin-on is supported by the Chulalongkorn University Dutsadi Phiphat Scholarship, Chulalongkorn University.

\section{REFERENCES}

1. Zhou X, Guo H, Chen K, Cheng H, Zhou R (2010) Identification, chromosomal mapping and conserved synteny of porcine Argonaute family of genes. Genetica 138, 805-12.

2. Sasaki T, Shiohama A, Minoshima S, Shimizu N (2003) Identification of eight members of the Argonaute family in the human genome. Genomics 82, 323-30.

3. Aporntewan C, Pin-on P, Chaiyaratana N, Pongpanich M, Boonyaratanakornkit V, Mutirangura A (2013) Upstream mononucleotide A-repeats play a cis-regulatory role in mammals through the DICER1 and Ago proteins. Nucleic Acids Res 41, 8872-85.

4. Wei KF, Wu LJ, Chen J, Chen Yf Xie Dx (2012) Structural evolution and functional diversification analyses of argonaute protein. J Cell Biochem 113, 2576-85.

5. Li W, Liu M, Feng Y, Xu Y-F, Che J-P, Wang G-C, Zheng J-H, Gao H-J (2013) Evaluation of Argonaute protein as a predictive marker for human clear cell renal cell carcinoma. Int J Clin Exp Pathol 6, 1086-94.

6. Chen Z, Lai T-C, Jan Y-H, Lin F-M, Wang W-C, Xiao H, Wang Y-T, Sun W, et al (2013) Hypoxia-responsive miRNAs target argonaute 1 to promote angiogenesis. J Clin Investig 123, 1057-67.

7. Li L, Yu C, Gao H, Li Y (2010) Argonaute proteins: potential biomarkers for human colon cancer. $B M C$ Canc 10, 38.

8. Yoo NJ, Hur SY, Kim MS, Lee JY, Lee SH (2010) Immunohistochemical analysis of RNA-induced silencing complex-related proteins AGO2 and TNRC6A in prostate and esophageal cancers. APMIS 118, 271-6.

9. Volpe TA, Kidner C, Hall IM, Teng G, Grewal SI, Martienssen RA (2002) Regulation of heterochromatic silencing and histone H3 lysine-9 methylation by RNAi. Science 297, 1833-7.

10. Pal-Bhadra M, Leibovitch BA, Gandhi SG, Rao M, Bhadra U, Birchler JA, Elgin SC (2004) Heterochromatic silencing and HP1 localization in Drosophila are dependent on the RNAi machinery. Science $\mathbf{3 0 3}$, 669-72.

11. Jones L, Keining T, Eamens A, Vaistij FE (2006) Virusinduced gene silencing of argonaute genes in Nicotiana benthamiana demonstrates that extensive systemic silencing requires Argonaute1-like and Argonaute4-like genes. Plant Physiol 141, 598-606.
12. Braun JE, Huntzinger E, Izaurralde E (2013) The role of GW182 proteins in miRNA-mediated gene silencing. In: Chan EKL, Fritzler MJ (eds) Ten Years of Progress in GW/P Body Research, Springer, New York pp 147-63.

13. Chen S, Chahar HS, Abraham S, Wu H, Pierson TC, Wang XA, Manjunath N (2011) Ago-2-mediated slicer activity is essential for anti-flaviviral efficacy of RNAi. PLoS ONE 6, e27551.

14. Shekar PC, Naim A, Sarathi DP, Kumar S (2011) Argonaute-2-null embryonic stem cells are retarded in self-renewal and differentiation. $J$ Biosci 36, 649-57.

15. Wassenegger M (2000) RNA-directed DNA methylation. In: Matzke MA, Matzke AJM (eds) Plant Gene Silencing. Springer, The Netherlands, pp 83-100.

16. Aporntewan C, Phokaew C, Piriyapongsa J, Ngamphiw C, Ittiwut C, Tongsima S, Mutirangura A (2011) Hypomethylation of intragenic LINE-1 represses transcription cancer cells through AGO2. PLoS ONE 6, e17934.

17. Wanichnopparat W, Suwanwongse K, Pin-on P, Aporntewan C, Mutirangura A (2013) Genes associated with the cis-regulatory functions of intragenic LINE-1 elements. BMC Genom 14, 205.

18. Aporntewan C, Mutirangura A (2011) Connection upand down-regulation expression analysis of microarrays (CU-DREAM): a physiogenomic discovery tool. Asian Biomed 5, 257-62.

19. Chalitchagorn K, Shuangshoti S, Hourpai N, Kongruttanachok N, Tangkijvanich P, Thong-ngam D, Voravud N, Sriuranpong V, et al (2004) Distinctive pattern of LINE-1 methylation level in normal tissues and the association with carcinogenesis. Oncogene 23, 8841-6.

20. Kuo MH, Allis CD (1999) Cross-linking and immunoprecipitation for studying dynamic protein: DNA associations in a chromatin environment. Methods 19, 425-33.

21. Schmitter D, Filkowski J, Sewer A, Pillai RS, Oakeley EJ, Zavolan M, Svoboda P, Filipowicz W (2006) Effects of dicer and Argonaute down-regulation on mRNA levels in human HEK293 cells. Nucleic Acids Res 34, 4801-15. 